Article

\title{
Is Humanity Doomed? Insights from Astrobiology
}

\author{
Seth D. Baum ${ }^{1,2}$
}

1 Department of Geography, Pennsylvania State University, University Park, PA 16802, USA;

E-Mail: sbaum@psu.edu; Tel.: 001-814-865-3433; Fax: 001-814-863-7943

2 Rock Ethics Institute, Pennsylvania State University, University Park, PA 16802, USA

Received: 19 January 2010 / Accepted: 8 February 2010 / Published: 12 February 2010

\begin{abstract}
Astrobiology, the study of life in the universe, offers profound insights into human sustainability. However, astrobiology is commonly neglected in sustainability research. This paper develops three topics connecting astrobiology to sustainability: constraints on what zones in the universe are habitable, the absence of observations of extraterrestrial civilizations, and the physical fate of the universe. These topics have major implications for our thinking and action on sustainability. While we may not be doomed, we must take certain actions to sustain ourselves in this universe. The topics also suggest that our current sustainability efforts may be of literally galactic importance.
\end{abstract}

Keywords: astrobiology; sustainability; environmental determinism; Fermi Paradox; physical eschatology; existential risk; global catastrophic risk; space colonization

\section{Introduction}

Is humanity doomed? In other words, is the current human population sustainable? This is among the most important questions we can ask today. And we are asking it—and trying to answer it—quite often, as the growing body of literature on sustainability indicates. The simple answer to this question is that we don't know. While there are pessimists who predict doom and optimists who deny this possibility, a more reasoned analysis suggests that our civilization's fate could go either way. Therefore, we must look around for clues that may help us unravel this great mystery.

In almost all cases, we look only to Earth for answers to this question. To an extent, this terrestrial focus is understandable. After all, Earth is our home planet and the place where we've spent all but a few brief moments of our existence to date. Furthermore, key sustainability challenges are, to a large extent, a product of our interactions with Earth's environment: climate change, biodiversity loss, and 
nitrogen and phosphorous cycle disruptions, among others [1]. Indeed, if our studies of sustainability did not focus heavily on Earth, we would be making a severe tactical error.

While a focus on Earth is important, deep insights about our sustainability are nonetheless to be found elsewhere. Earth is not a closed system, and its interactions with the rest of the universe strongly affect our sustainability. Through studying the physical and biological makeup of the rest of the universe, we can appreciate our own place-and our duration-within it. This exercise is a natural extension of much terrestrial sustainability research, which exhorts us to recognize and act on connections between our own lives and phenomena across broader spatial and temporal scales. Thus consideration of phenomena beyond Earth is readily compatible with existing sustainability paradigms.

The study of life in the universe is the focus of the emerging discipline of astrobiology. The NASA Astrobiology Institute defines astrobiology as "the study of the origin, evolution, distribution, and future of life in the universe" [2]. Astrobiology studies both life on Earth and possible life elsewhere in the universe. Astrobiology, like sustainability, is a transdisciplinary field, meaning that it transgresses disciplinary boundaries, drawing on whatever information and methods best help answer the questions it asks. Astrobiology can potentially span each of the natural and social sciences, humanities, and engineering. However, as it is currently practiced, it focuses predominantly on the natural sciences, in particular astronomy, biology, chemistry, and geology.

This paper develops several key ways in which astrobiology informs the study of human sustainability. Three topics are covered. The first topic, environmental determinism, informs the question of whether humanity's fate is determined by its environment or is instead dictated by human activity. The second topic, the Fermi Paradox, uses the absence of observations of extraterrestrial civilizations to inform the questions of what is at stake with current human sustainability efforts and how likely those efforts are to succeed. The third topic, physical eschatology, informs the question of whether the human population can be sustained indefinitely and what this possibility means for us now. While these astrobiology topics do not definitively answer the question of whether humanity is doomed, they do bring significant insights and also demonstrate that consideration of life elsewhere is of profound importance to the study of sustainability on Earth.

\section{Environmental Determinism}

Environmental determinism is the idea that outcomes of human societies are determined by the environments in which humans live instead by human activity and initiative. If outcomes are determined by environments, then there may be little we can do to sustain ourselves. Either our success at sustainability is guaranteed by certain properties of our environment, or we are doomed. Alternatively, if outcomes are not determined by environments, then our fate may lie in our own hands. In this case our efforts towards achieving sustainability may be crucial to our success. Thus whether or not environments determine outcomes is of great importance to sustainability.

Many generations of scholars have considered environmental determinism. Early 20th century scholars, particularly in the field of geography, gave environmental explanations for Europeans' colonial successes and even the perceived physiological and cultural superiority of European people relative to people elsewhere. This form of environmental determinism supported attitudes and practices 
that are today broadly rejected, such as racism, colonialism, and slavery. It was claimed, for example, that warm weather made people in the tropics inherently lazy, whereas colder climates caused people to be more industrious. Europeans used this perceived superiority as grounds for the colonization and exploitation of others [3].

This crude environmental determinism is now broadly rejected on both empirical and moral grounds. While environments do strongly influence some aspects of human physiology (in particular skin color), it is now clear that humans from all regions are highly capable of success. Meanwhile, moral principles of fairness and equality compel us to treat each other with a respect and dignity incompatible with the institutions of colonization and slavery. A more nuanced understanding of the role of environments in our lives is warranted.

There is much ongoing debate about the extent to which human outcomes are determined by environmental factors. Jared Diamond's popular book Guns, Germs, and Steel [4] argues that while humans from all regions are highly capable, some regions had better conditions for successful civilization, and that this largely explains broad patterns of human history. For example, Eurasia's large number of domesticable plants and animals allowed a strong agriculture to develop early on, which contributed to the development of dominant civilizations. Other scholars, such as James Blaut, have criticized this work, questioning its empirical accuracy and claiming it underestimates the extent to which human decisions define outcomes [5,6]. While it is clear that the environment has some influence (for example, some regions really did lack domesticable animals), exactly what this influence is remains an open question.

The implications of environmental determinism for sustainability are profound. If human outcomes are determined primarily by environmental factors, then there may be little we can do to sustain our civilization. This pessimistic, collapse-is-inevitable attitude dates to at least Thomas Malthus [7] and has contemporary support from scholars including Anne and Paul Ehrlich [8] and also Jared Diamond, as seen in the latter's book Collapse [9], though it is important to note that each of these scholars has proposed ways in which humans might be able to avoid collapse. Alternatively, if human outcomes are determined primarily by human decision making, then there is much we can (and likely will) do to sustain our civilization. This optimistic, collapse-is-avoidable attitude dates to at least Ester Böserup [10] and has contemporary support from scholars such as Julian Simon [11]. While it is clear that human capabilities have limits (for example, we cannot create new energy, except through mass-energy conversions), exactly what these limits are is another open question.

The questions of environmental determinism and its implications are strongly informed by astrobiology. Consider first the question of the extent to which human outcomes are determined by environments. As Jared Diamond points out, some regions on Earth have had better conditions for success than others. Through astrobiology, we understand that Earth itself has better conditions for success than some places - and potentially worse conditions than other places. It is the only place in the solar system to have hosted an immense flourishing of life, though other places such as Mars and perhaps also Jupiter's moon Europa may prove to have hosted small quantities of life too. The reason for this is simple: Earth is in a habitable zone, i.e., a region in space and time which is capable of being inhabited. Location within a habitable zone determines that it is possible for life and civilization to exist, although it does not guarantee that life and civilization will come to exist. 
Habitable zones exist across many scales. The early universe lacked stars and planets, as do the vast regions between galaxies and, within galaxies, between star systems. Life as we know it could not exist in these times and places. Life as we know it could also not exist within certain zones of star systems. Some zones are too hot (too close to the stars) whereas others are too cold (too far from the stars) for liquid water to exist on the surface of a planet [12]. The idea that only the intermediate zones are habitable is known as the Goldilocks principle. The principle applies to galaxies as well. The spatial centers of galaxies have too many supernovae, bringing too much radiation; stars at the spatial edges have too few heavy elements to form planets [13]. Earth is in a zone that is, simply put, just right.

We can also speak of habitable zones within Earth. These zones are broad but nonetheless limited. The portions of Earth that are not habitable are still within habitable zones at the scale of the galaxy and the solar system, but they are not within habitable zones at the scale of the planet. For example, life may not have been able to exist in the earliest Earth atmospheres, or in the Earth's hot, dense core. We now know that life can nonetheless exist in a surprisingly broad range of Earth environments, including some very extreme ones. The extremophiles who live in extreme environments are of great interest to astrobiologists for the insights they offer into the breadth of situations in which life can exist. Meanwhile, advanced civilization exists in a narrower zone on Earth. Extremophiles tend to be singlecelled organisms incapable of anything similar to the scientific, cultural, and other achievements of humanity. Also, perhaps civilizations like ours could not form in aquatic environments, even though these environments can support a rich diversity of complex and even very intelligent organisms. It is thus clear that humanity at large benefits from "geographic luck" that many other species never had, and that many other zones of the universe couldn't have. We can thus speak of an astrobiological environmental determinism: location within a habitable zone determines whether or not life or civilization could exist.

The implications that this astrobiological environmental determinism has for human sustainability are more complex. Civilizations (human or otherwise) can only exist within certain habitable zones, but once a civilization does exist, it does not immediately follow that the civilization will necessarily succeed or fail. In other words, recognizing this astrobiological environmental determinism does not commit us to the pessimism of Malthus, Ehrlich, and Diamond, nor does it guarantee us the optimism of Böserup and Simon. Further inquiry is needed. Some of this inquiry is the Earth-oriented study that makes up the bulk of sustainability research. But further information can be found from astrobiology. We now turn to insights found from the possibility of civilizations originating in places other than Earth.

\section{The Fermi Paradox}

Simple, back-of-the-envelope calculations suggest that the galaxy should be full of intelligent civilizations. The calculations use such factors as the age and size of the galaxy, the numbers of stars and planets, the portions of planets in habitable zones, and the distances between planets and star systems. The paradox that follows from this calculation, initially performed by physicist Enrico Fermi, is: If we expect civilizations to appear throughout the galaxy, then where are they? 
Over the several decades since Fermi developed this paradox, many solutions have been proposed and developed by astrobiologists and others [14]. We know sufficiently little about the galaxy that we cannot rule many, or perhaps even any, of these solutions out. But some solutions nonetheless seem more plausible, and some solutions have more significant implications for us here on Earth. An example of a solution that has less significance is the possibility that extraterrestrial civilizations exist but are hiding from us so that they can observe us for entertainment, like a galactic version of the film The Truman Show. Strictly speaking, we cannot rule out this solution, but it seems unlikely and there's also not much we can do about it. Meanwhile, several other solutions are quite plausible and have major implications for us Earthlings, including for our sustainability.

Perhaps the most frightening solution to the Fermi Paradox is the possibility that all civilizations inevitably destroy themselves soon after they become sufficiently intelligent and capable of doing so. This solution gained popularity during the Cold War era, when concern about nuclear warfare was very high. While Cold War tensions have cooled, our ongoing experience on Earth is such that this solution remains quite plausible. If this solution does explain the absence of observations of extraterrestrial civilizations, then our own future has a very ominous outlook. If intelligent civilizations elsewhere in the galaxy destroy themselves before colonizing the galaxy, then our own civilization will likely suffer the same fate. Given that we are now gaining the capacity for self-destruction, we should hope that that is not the inevitable fate of civilizations in our galaxy. To be sure, even if all other civilizations in the galaxy destroyed themselves, it remains possible that we could succeed where they failed. But such success must be viewed as highly unlikely. This bodes poorly for humanity's chances of achieving sustainability. Still, perhaps we will succeed where others have failed, and perhaps our current sustainability efforts will permit that to happen.

Another very plausible solution has recently been developed, drawing directly from humanity's ongoing struggles with sustainability on Earth [15]. This sustainability solution is considerably more optimistic than the inevitable-destruction solution discussed above. The sustainability solution revisits an assumption in the basic calculation suggesting that civilizations should be widespread in the galaxy. This assumption is that civilizations will expand exponentially throughout the galaxy. Exponential growth is a phenomenon observed widely (but not universally) on Earth across both human and nonhuman populations, which explains why it was assumed in the calculation. However, as we now know all too well (but didn't know as well when the calculation was originally developed), when a population expands exponentially, it often bumps into resource constraints and related sustainability issues. In short, exponential growth is often unsustainable. This observation can explain the absence of observations of extraterrestrial civilizations.

When a population expands exponentially, it typically experiences one of two fates. First, it could overshoot the carrying capacity of the ecosystem supporting it, depleting key resources. In this scenario, the population quickly suffers a dramatic crash. The damage to the population or to the ecosystem is often permanent such that the population never regrows its numbers. Perhaps extraterrestrial civilizations that continue to expand exponentially suffer similar crashes before they can expand throughout the galaxy, or before they would be observed by us or any other civilization. While civilizations sufficiently intelligent for space travel might understand the dangers of unsustainable expansion, they might not act on this understanding, just as human civilization does not 
always act on its own understanding of these dangers. This would explain why we do not observe other civilizations.

The other fate of an exponentially expanding population avoids the crash. In this scenario, the population slows and possibly ceases its growth early enough that it remains within the carrying capacity of its ecosystem. This growth pattern approximates a logistic curve and is also commonly observed in populations on Earth. This growth pattern is fundamentally sustainable. We may be experiencing this growth pattern with human populations, since global human population growth has been slowing in recent decades. In this case, the reason we do not observe extraterrestrial civilizations is because they do not expand rapidly enough to fill the galaxy. These civilizations could be those that understood the dangers of unsustainable expansion and successfully acted on this understanding. They are out there, but they are hard to find.

Note that some extraterrestrial civilizations might not expand at all. They might be highly intelligent but simply not desire to expand. Indeed, there are human populations that do not pursue expansion but instead favor other objectives. The existence of intelligent, non-expansive extraterrestrial civilizations is fully compatible with the sustainability solution to the Fermi Paradox because these civilizations do not expand rapidly-indeed, they do not expand at all. If the sustainability solution explains the absence of observation of extraterrestrial civilizations, then non-expansive civilizations and slowlyexpanding civilizations could exist. Both of these civilization types would be sustainable, but they would also be hard for us to find.

How extraterrestrials respond to their sustainability challenges is informed by our previous discussion of environmental determinism. If it is the case in general that environments do or don't determine civilizational outcomes, then this would apply to extraterrestrial civilizations just as it applies to human civilizations. If environments do determine outcomes, then all civilizations could fail to achieve sustainability, regardless of whether they understand the dangers of unsustainable expansion. Alternatively, if environments do not determine outcomes, then some (but not necessarily all) civilizations could successfully achieve sustainability, thereby avoiding a crash.

The connection between environmental determinism and extraterrestrial sustainability implies a connection between sustainability on Earth and the search for extraterrestrial intelligence (SETI). If humanity successfully achieves sustainability, then we will know that sustainability is possible in our galaxy. While extraterrestrial civilizations would not necessarily achieve sustainability, it becomes more likely that they would. We may be able to detect these civilizations. Further detail on the implications of the sustainability solution to SETI are discussed elsewhere [15].

The sustainability solution also has important implications for our sustainability efforts here on Earth. If this is why we do not observe extraterrestrials (and it of course might not be), then it follows that exponential growth cannot be sustained at the galactic scale. Either we must slow our own growth rate so that we stay within the carrying capacities of our ecosystems, or we are doomed to suffer a major population crash. Some caution is warranted here. It may be that the sustainability issues don't become important until a civilization expands to an interplanetary or interstellar scale. If this is the case, then humanity could potentially sustain exponential growth until it has grown beyond Earth. This case would mean that our present concerns about sustainability on Earth might not matter. However, it still means that humanity will need to make an eventual transition to sustainability, and thus that our 
current efforts may set us in that direction, thereby avoiding an eventual crash. And it is also possible that the sustainability issues for civilizations across the galaxy become important at the planetary scale. In this case, our current sustainability efforts may be crucial to us avoiding a crash. One way or another, the possibility that sustainability resolves the Fermi Paradox increases the importance of our current efforts to live sustainably on Earth.

There is one additional Fermi Paradox solution worth discussing in the context of sustainability. That is the simple possibility that we here on Earth are the only life, or the only intelligent life, anywhere in the galaxy. Today there are several astrobiologists who consider this solution to be likely [16]. If this solution is correct, then we learn nothing new about our own future prospects, except that we can breathe a sigh of relief that our self-destruction or population crash is not inevitable. However, if this solution is correct, then it makes our activities here on Earth that much more important, because we then become the galaxy's (and perhaps the universe's) only shot at something truly special. It is up to us to decide what that special something should be, and to make it happen. Major insight into these matters can be found by considering the fate of the world and the universe(s).

\section{Physical Eschatology}

Eschatology is the study of the end times, whether it's the end of the world, of the universe(s), or of humanity, such as in the apocalypse. Eschatology is commonly studied as a philosophical or religious topic; religious traditions vary on questions such as whether the end is near and whether it is desirable. The term physical eschatology has been developed to refer to the study of the end of physical phenomena such as the Earth, the solar system, and the universe [17]. While the beginning of the universe has historically attracted more attention from physicists (perhaps because more data is available), physical eschatology has received some focused attention. From this, some initial conclusions can be drawn. These conclusions, and their very direct significance for sustainability, are discussed here.

Above, we established that civilization can only exist within habitable zones. If our concern is with the sustainability of human civilization, then we must ask the question of how long human civilization can exist within habitable zones. Some answers to this question do not require astrobiology. For example, a nuclear war or ecological collapse could render Earth not habitable to humans, though in these scenarios the planet would likely remain habitable for other organisms. But other answers depend heavily on insights from astrobiology. We will consider the scenarios of these other answers in chronological order.

The earliest scenario is the impact event: collision between an asteroid or comet and Earth. Such collisions are relatively commonplace, but with impactors too small to prevent human civilization from being sustained. It is possible for small impactors to cause outsized damage if they land in the wrong place. For example, a 2002 asteroid exploded over the Mediterranean; had it landed a few hours earlier, it would have exploded over Kashmir. A Kashmir impact plausibly could have initiated a nuclear exchange between India, Pakistan, and possibly others [18]. Larger, less-common asteroids could land anywhere on the planet and still cause major damage. Such asteroids are a major threat to human sustainability [19]. Indeed, a large asteroid is believed to have caused at least one major extinction 
event, the Cretaceous-Tertiary. Several major space agencies, including those of Russia and the United States, have active programs to monitor the skies for threatening asteroids. Recently the Russian agency has proposed-to some controversy-deflecting an asteroid that has a small but non-zero chance of hitting Earth in 2029 [20]. Regardless of what the merits of this proposal might be, it is clear that an active asteroid deflection program could play an important role in sustaining life and civilization on Earth.

The next scenario is the end of the world. The idea that the world will end is quite often neglected in discussions of sustainability, as is apparent from the several definitions of sustainability advocating efforts along the lines "indefinite" survival into the "distant future" here on Earth [21]. Despite this neglect from sustainability researchers, the end of the world is actually a fairly well-understood phenomenon. Earth's end is driven by two changes in the Sun: an increase in outgoing radiation and an increase in size. In several hundred million years, increased radiation could warm the planet, causing greater silicate rock weathering and lowering atmospheric $\mathrm{CO}_{2}$ levels below 10 parts per million, at which point $\mathrm{C} 4$ photosynthesis could not occur. C3 photosynthesis requires at least 150 parts per million $\mathrm{CO}_{2}$ and thus would cease earlier [22]. No contemporary species-except a few extremophiles - could survive in these conditions. Human civilization certainly could not be sustained.

If our civilization remains intact at that point, then we would have one option that would let us remain on Earth: blocking some of the incoming radiation. Such a project would resemble some of the geoengineering schemes currently under consideration as a possible response to the more immediate global warming Earth is experiencing due to anthropogenic greenhouse gas emissions. Other geoengineering schemes, such as adjusting Earth's albedo, may also work here. If we can implement such geoengineering as the sun's radiative output increases, then we-and other contemporary species - could continue to inhabit Earth [23].

Geoengineering could extend Earth's habitable lifetime beyond the next several hundred million years, but, in all likelihood, it cannot extend this lifetime forever. In several billion years, the sun will expand in size, eventually engulfing Earth and other planets. The resulting temperatures at the Earth's surface would be devastating. It is very difficult to imagine any geoengineering techniques or other scenarios that could keep Earth habitable then [24,25], unless it somehow becomes feasible to alter Earth's orbital radius. Of course, our geoengineering knowledge could change radically in the next billion or so years, so such techniques cannot be strictly ruled out today. However, given contemporary knowledge, our best guess is that in a few billion years, life on Earth will cease to be habitable.

But just because Earth cannot sustain us forever, it does not follow that we cannot be sustained forever, i.e., that we are doomed. It remains possible that we could live on elsewhere in the universe. Already, humanity has taken some important steps towards space colonization, including manned and unmanned space flight and related scientific and engineering activities. Given this early success, we must have a reasonable likelihood of being able to achieve a sustainable space colonization program sometime before the world ends in a billion or so years. If self-sustaining space colonies can be achieved, then we can survive for longer than our home planet. Indeed, we can survive until the rest of the universe ceases to be habitable.

The fate of the universe is understood much less well than the fate of the world. The physics simply remains too unsettled. There are several competing theories $[25,26]$. In about $10^{14}$ years, stars stop 
shining. Perhaps we will be unable to survive without stellar radiation. If we can survive, then in about $10^{32}$ years, a significant portion of protons may have decayed. While we may be able to live without Earth or even without stars, we probably cannot live without protons. Other theories suggest that the universe may remain habitable forever [27], or that we may be able to wormhole to a different universe before our home universe ends [28]. Some of these theories may seem more plausible than others today, but it is hard to rule any of them out. Our understanding of the relevant physics can change a lot in the next $10^{14}$ years or so.

The possibility that the universe(s) may remain habitable forever is of significance to some contemporary ethical and decision making theory. These theories are those that say we should do whatever maximizes the total amount of some form of value; in philosophical terms, this is consequentialist ethics. This value can be human welfare, or the welfare of all sentient animals, or the fitness of ecosystems, or life itself. For the fate of the universe to matter, the theories must also place some value on what happens in the distant future, and they must handle uncertainty through something along the lines of an expected value. Then, the greater-than-zero probability that the universe may remain habitable forever means that the expected value of many decisions will be infinite. Infinite expected values pose significant theoretical challenges, which much research has attempted to resolve [29-31]. The astrobiology of the fate of the universe means that these theoretical challenges are potentially of actual practical relevance to decisions we could face.

For today's decisions, it is less important which theory of the fate of the universe turns out to be correct. This is because all the theories agree on one crucial point: the universe can remain habitable for many orders of magnitude longer than Earth can. The billion or so years we can remain on Earth is, from a universal perspective, the short-term. Long-term sustainability requires space colonization. If space colonization can be achieved, then Earth-originating civilization (or life, or whatever else we might care about) can be sustained for a much, much longer period of time. In short, almost all of the opportunity for life or civilization is beyond Earth. This conclusion should be a major feature in our thinking about sustainability.

Should we care about sustaining civilization into the long-term, distant future? This is fundamentally an ethical question. Many sustainability advocates clearly do care about long-term sustainability. I agree with them. I see no compelling reason why we should value something more based strictly on when it occurs, just as I see no compelling reason why we should value something more based on where it occurs, or its race, or its gender, or its species. All these concerns strike me as morally irrelevant. I am hardly alone in holding this view about the moral irrelevance of time [32-34], which follows from very basic principles of equality. But others disagree, and there is vigorous debate on them, often under the rubric of discounting [35-37]. Further discussion of this debate is beyond the scope of this article; for the remaining discussion, I will simply assume that we care about long-term sustainability. If nothing else, the possibility of long-term sustainability can be of intellectual or scientific interest.

The fact that the universe will remain habitable for much longer than Earth will means that, if we care about long-term sustainability, then it is extremely important for us to colonize space [38]. Colonizing space will permit us to take advantage of all that the rest of the universe has to offer [39]. But this does not mean that we should focus our current efforts on space colonization. The reason for 
this is simple: Earth will remain habitable for another billion years or so. While a billion years is quite small compared to the universe's lifetime, it is quite large compared to the amount of time it probably takes to colonize space, especially given our current rapid rates of technological change. If we are to colonize space before the world ends, then we have plenty of time to do it - as long as nothing really bad happens first.

These "really bad" things can be any global catastrophe so large that it would permanently eliminate our capacity to colonize space before the world ends. Several phenomena may be so catastrophic, including nuclear warfare, pandemic outbreaks, ecological collapse, disruptive technology, and of course impact from a large asteroid. Risks of these events have been called global catastrophic risks or existential risks [40]. I will use the term existential risk here because it is our existence that is ultimately at stake. These risks are far more imminent than the end of the world. Therefore, if we care about long-term sustainability, then we should focus our efforts on avoiding these catastrophes, i.e., on reducing existential risk, so that future generations can colonize space.

A focus on existential risk reduction will in some cases require a shift of focus for those working on sustainability. Much contemporary sustainability work helps to reduce existential risk, but some of it is more effective than others, and some other effective opportunities go overlooked. Sustainability work focused on more local concerns may not make much difference to the broader course of civilizationalthough much caution is warranted here, because, as sustainability researchers know well, local changes can often have universal consequences. Nonetheless, those who care about long-term sustainability should seek out opportunities to reduce existential risk with an eye towards eventual space colonization.

It is worth noting that space colonization itself can help with short-term sustainability. If a catastrophic event causes the extinction of humans on Earth, then a space colony may be our only hope of survival. Stephen Hawking is among those calling for short-term space colonization for this reason [41]. Similarly, if a catastrophic event significantly harms but does not eliminate humanity's presence on Earth, then a space colony can be used to help revive civilization on Earth. One recent proposal calls for installing a comprehensive digital library on the moon which would help a post-catastrophe civilization get back on its feet [18]. This proposal parallels calls for refuges [42] or seed banks [43] on Earth.

Astrobiology has central roles to play in all of the ways that physical eschatology is relevant to sustainability. Astrobiology helps us understand how and when the world and the universe(s) could become not habitable. Indeed, without astrobiology, we would overlook the very possibility that the world and universe(s) could become not habitable in the first place. Astrobiology also helps us understand what our response options are, and helps us focus our current attention on existential risk reduction. These conclusions have very major implications for how we should think about and practice sustainability on Earth today.

\section{Conclusion}

Is humanity doomed, or is it instead sustainable? At present, we do not know. This paper has developed several major ways in which astrobiology informs our answers to this question. 
Astrobiology informs us that life can only be sustained in habitable zones, and that this implies that some aspects of our civilization are strictly determined by our environment. Astrobiology also informs us that there are several explanations for why we have never observed any extraterrestrial civilizations, including one explanation derived from sustainability on Earth, and that the absence of extraterrestrials gives our current sustainability efforts a literally galactic significance. Finally, astrobiology informs us that Earth's environment will not remain habitable forever, but that the universe just might, or at least will remain habitable for much, much longer. This suggests the importance of colonizing space before the world ends, which in turn suggests the importance of avoiding the global catastrophes that threaten our civilization's existence today.

For these reasons, astrobiology is of profound importance to the study of sustainability on Earth. In order for us to benefit from the rich insights astrobiology has for sustainability, we should give astrobiology an important place in the study of sustainability. Forming connections between astrobiology and sustainability research can be fairly straightforward. Both fields are already accustomed to transdisciplinary research, and to embracing a large diversity of intellectual perspectives. Furthermore, both fields ask very big-picture questions about humanity's place in the grander scheme of things. In many ways, these fields are two sides of the same coin, with one focused on life on Earth and the other focused on life in the universe. Hopefully these fields will successfully learn from each other and progress together. The stakes could not be any greater.

\section{Acknowledgements}

Jacob Haqq-Misra, Gilman Ouellette, Shawn Domagal-Goldman, and three anonymous reviewers provided very helpful comments on earlier versions of this article.

\section{References and Notes}

1. Rockström, J.; Steffen, W.; Noone, K.; Persson, Å.; Chapin, F.S., III; Lambin, E.F.; Lenton, T.M.; Scheffer, M.; Folke, C.; Schellnhuber, H.J.; Nykvist, B.; de Wit, C.A.; Hughes, T.; van der Leeuw, S.; Rodhe, H.; Sörlin, S.; Snyder, P.K.; Costanza, R.; Svedin, U.; Falkenmark, M.; Karlberg, L.; Corell, R.W.; Fabry, V.J.; Hansen, J.; Walker, B.; Liverman, D.; Richardson, K.; Crutzen, P.; Foley, J.A. A safe operating space for humanity. Nature 2009, 461, 472-475.

2. About Astrobiology; NASA Astrobiology Institute: Mountain View, CA, USA, 2008; Available online: http://astrobiology.nasa.gov/about-astrobiology (accessed on 12 January 2010).

3. Livingstone, D.N. The Geographical Tradition: Episodes in the History of a Contested Enterprise; Blackwell: Oxford, UK, 1992.

4. Diamond, J. Guns, Germs, and Steel: The Fates of Human Societies; Norton: New York, NY, USA, 1997.

5. Blaut, J.M. Eight Eurocentric Historians; Guilford Press: New York, NY, USA, 2000.

6. Merrett, C.D. Debating destiny: Nihilism or hope in guns, germs, and steel? Antipode 2003, 35, 801-806.

7. Malthus, T.R. An Essay on the Principle of Population; J. Johnson: London, UK, 1798. 
8. Ehrlich, A.; Ehrlich, P. The population bomb revisited. Electron. J. Sustain. Dev. 2009, 1, 63-71.

9. Diamond, J. Collapse: How Societies Choose to Fail or Succeed; Viking: New York, NY, USA, 2005.

10. Böserup, E. The Conditions of Agricultural Growth: The Economics of Agrarian Change under Population Pressure; Aldine: Chicago, IL, USA, 1965.

11. Simon, J. The Ultimate Resource 2; Princeton University Press: Princeton, NJ, USA, 1998.

12. Kasting, J.F.; Whitmire, D.P.; Reynolds, R.T. Habitable zones around main sequence stars. Icarus 1993, 101, 108-128.

13. Lineweaver, C.H.; Fenner, Y.; Gibson, B.K. The galactic habitable zone and the age distribution of complex life in the milky way. Science 2004, 303, 59-62.

14. Webb, S. If the Universe Is Teeming with Aliens... Where is Everybody? Fifty Solutions to the Fermi Paradox and the Problem of Extraterrestrial Life; Copernicus Books: New York, NY, USA, 2002.

15. Haqq-Misra, J.D.; Baum, S.D. The "sustainability solution" to the Fermi Paradox. J. $B r$. Interplanet. Soc. 2009, 62, 47-51.

16. Ward, P.D.; Brownlee, D. Rare Earth: Why Complex Life Is Uncommon in the Universe; Copernicus Books: New York, NY, USA, 2000.

17. Ćirković, M. Resource letter PEs-1: Physical eschatology. Am. J. Phys. 2003, 71, 122-133.

18. Burrows, W.E. The Survival Imperative; Tom Doherty: New York, NY, USA, 2006.

19. Napier, W. Hazards from Comets and Asteroids. In Global Catastrophic Risks; Bostrom, N., Ćirković, M., Eds.; Oxford University Press: Oxford, UK, 2008; pp. 222-237.

20. Barry, E. Russia to plan deflection of asteroid from Earth. New York Times, 30 December 2009; Available online: http://www.nytimes.com/2009/12/31/world/europe/31asteroid.html (accessed on 8 January 2010).

21. Pezzey, J. Sustainable Development Concepts: An Economic Analysis; World Bank Environment Paper Number 2; The World Bank: Washington, DC, USA, 1992.

22. Caldeira, K.; Kasting, J. The life span of the biosphere revisited. Nature 1992, 360, 721-723.

23. Baum, S.D. Cost-Benefit analysis of space exploration: Some ethical considerations. Space Policy 2009, 25, 75-80.

24. Laughlin, G.; Adams, F.C. The frozen Earth: Binary scattering events and the fate of the solar system. Icarus 2000, 145, 614-627.

25. Adams, F.C. Long-Term astrophysical processes. In Global Catastrophic Risks; Bostrom, N., Ćirković, M., Eds.; Oxford University Press: Oxford, UK, 2008; pp. 33-47.

26. Adams, F.; Laughlin, G. A dying universe: The long-term fate and evolution of astrophysical objects. Rev. Mod. Phys. 1997, 69, 337-372.

27. Dyson, F.J. Time without end: Physics and biology in an open universe. Rev. Mod. Phys. 1979, 51, 447-460.

28. Kaku, M. Parallel Worlds: The Science of Alternative Universes and Our Future in the Cosmos; Penguin: London, UK, 2005. 
29. Bostrom, N. The Infinitarian Challenge to Aggregative Ethics; Faculty of Philosophy, Oxford University: Oxford, UK, 2008; Available online: http://www.nickbostrom.com/ethics/infinite.pdf (accessed on 12 January 2010).

30. Lauwers, L.; Vallentyne, P. Infinite utilitarianism: More is always better. Econ. Philos. 2004, 20, 307-330.

31. Zame, W. Can utilitarianism be operationalized? Theor. Econ. 2007, 2, 187-202.

32. Broome, J. Discounting the future. Philos. Public Aff. 1994, 23, 128-156.

33. Cowen, T.; Parfit, D. Against the social discount rate. In Justice between Age Groups and Generations; Laslett, P., Fishkin, J., Eds.; Yale University Press: New Haven, CT, USA, 1992; pp. 144-161.

34. Caney, S. Human rights, climate change, and discounting. Environ. Polit. 2008, 17, 536-555.

35. Discounting and Intergenerational Equity; Portney, P., Weyant, J., Eds.; Resources for the Future: Washington, DC, USA, 1999.

36. Weisbach, D.A.; Sunstein, C.R. Introduction: symposium on intergenerational equity and discounting. Univ. Chic. Law Rev. 2007, 74, 1-3.

37. Zeckhauser, R.J.; Viscusi, W.K. Discounting dilemmas: editors' introduction. J. Risk Uncertain. 2008, 37, 95-106.

38. Tonn, B.E. Distant futures and the environment. Futures 2002, 34, 117-132.

39. Bostrom, N. Astronomical waste: The opportunity cost of delayed technological development. Utilitas 2003, 15, 308-314.

40. Global Catastrophic Risks; Bostrom, N., Ćirković, M., Eds.; Oxford University Press: Oxford, UK, 2008.

41. Move to new planet, says Hawking. BBC News, 30 November 2006; Available online: http://news.bbc.co.uk/1/hi/uk/6158855.stm (accessed on 12 January 2010).

42. Hanson R. Catastrophe, social collapse, and human extinction. In Global Catastrophic Risks; Bostrom, N., Ćirković, M., Eds.; Oxford University Press: Oxford, UK, 2008; pp. 363-377.

43. Charles, D.A. "Forever" seed bank takes root in the Arctic. Science 2006, 312, 1730-1731.

(C) 2010 by the authors; licensee Molecular Diversity Preservation International, Basel, Switzerland. This article is an open-access article distributed under the terms and conditions of the Creative Commons Attribution license (http://creativecommons.org/licenses/by/3.0/). 